\title{
A novel temporal trust-based recommender system
}

\begin{abstract}
While Collaborative Filtering $(\mathrm{CF})$ recommender systems, which focus on previous indicate preferences, are known for their traditional problems such as cold-start, sparsity and modest accuracy, trust-based CF has been previously proposed to solve such issues by focusing on trust values among the users. Nonetheless, most existing approaches use trust as an independent factor from time, in this paper we argue that trust value between users is dynamic; hence it change over time. For this reason we propose a novel temporal-trust based approach to calculate trust values aware of time of friendship. To validate the proposed approach in this paper, we used Delicious data set and compared our approach with traditional CF and trust-based approaches. Results showed that accuracy of proposed approach overcomes the traditional approaches.
\end{abstract}

Keyword: Component; Recommender systems; Collaborative filtering; Trust; Temporaltrust, Social networks 